\title{
Assessment of Agreement between Pretest Probability Score and Summed Stress Score of Myocardial Perfusion Imaging in Coronary Artery Disease
}

\author{
Sharmin Rahman $^{1}$, Mohammad Simoon Salekin ${ }_{1}^{2}$, Pupree Mutsuddy ${ }_{3}^{2}$, Rezoana Ahmed ${ }_{4}^{1}$, Tasnia Kawser Kanika ${ }^{1}$, Khaleda \\ Mushtary , Sadia Sultana ${ }^{3}$ and Raihan Hussain ${ }^{4}$ \\ 1 Ex-M.Phil Student, NINMAS, BSMMU, ${ }_{2}^{2}$ Senior Medical Officer, NINMAS, BSMMU, ${ }_{3}$ Professor \& Head, Nuclear Nephrology \\ Division, NINMAS, ${ }^{4}$ Ex-Member, Bio-Sciences Division, BAEC \\ Correspondence Address: Dr. Sharmin Rahman, Ex-M Phil (Nuclear Medicine) Student,
}

National Institute of Nuclear Medicine and Allied Sciences (NINMAS), e-mail : drsharminmadhuri@gmail.com

\begin{abstract}
Objective: Cardiovascular diseases are considered an important cause of mortality \& morbidity in many developing countries including Bangladesh. The first step in evaluating a patient with Coronary Artery Disease (CAD) is the clinical assessment of pretest probability. American Heart Association/ American College of Cardiology (AHA/ACC) guidelines recommend the use of Diamond and Forrester Method (DFM) or Duke Clinical Score (DCS) for calculating Pretest Probability Score (PPS). Myocardial Perfusion imaging (MPI) can calculate the Summed Stress Score (SSS), an index obtained by adding the individual scores derived from the 17 segments. This study was performed to assess the agreement between the established PPS with SSS so that it can help in risk stratification.
\end{abstract}

Patients and Methods: This cross-sectional observational study was carried out in National Institute of Nuclear Medicine \& Allied Science (NINMAS), BSMMU from July 2016 to June 2017. A total of 89 suspected or known CAD patients were included in this study. PPS was calculated by Duke clinical scoring from brief clinical history. SSS was calculated by nuclear medicine software while performing MPI. Statistical analyses was carried out by using the IBM Statistical Package for Social Sciences (SPSS) version 20.0.0 (IBM Corporation Software Group Somers, NY). Pearson correlation and Bland \& Altman analyses were applied for assessing correlation and agreement between PPS and SSS. Degree of relation between variables is expressed by ' $r$ ' (Pearson's correlation coefficient).

Results: The mean of PPS was found $14.73 \pm 3.35$ and that of SSS was found $16 \pm 14.01$. A positive correlation $(r=0.108 ; p=0.312)$ between PPS and SSS. With Bland and Altman analysis, it was observed that mean difference of PPS and SSS was $-1.27 \pm 14.045$. The limit of agreement ranged from -28.798 to 26.259 . There was a positive correlation between PPS and SSS. Mean difference between the two scores was small. The bias between the scores was not significant. The differences within mean $\pm 1.96 \mathrm{SD}$ were not statistically significant.

Conclusion: This study shows PPS and SSS can be used interchangeably. This analysis of agreement between PPS and SSS can further enhance prediction of CAD and upgrade the utilization of SSS for risk stratification in CAD patients, which will influence therapeutic management of the patients and play a major role to reduce cardiovascular mortality and morbidity.

Keywords: Pretest Probability Score, Summed Stress Score, Myocardial Perfusion Imaging, Coronary Artery Disease.

Bangladesh J. Nucl. Med. Vol. 21 No. 2 July 2018

Doi : https://doi.org/10.3329/bjnm.v21i2.40326

\section{INTRODUCTION}

Coronary Artery Disease (CAD) is the leading cause of death worldwide (1). CAD, also known as Ischemic Heart Disease (IHD), is a group of diseases that includes: stable angina, unstable angina, myocardial infarction, and sudden cardiac death (2). The age adjusted death rate is 53.53 per 100,000 of population and ranks Bangladesh at number 150 in the world (3).

National and international guidelines on the investigation of stable $\mathrm{CAD}$ have given increasing importance to the pretest probability for the presence of $\mathrm{CAD}$, provided risk scores and recommended the optimal investigation for the different risk score categories (4). One can estimate the pretest probability for CAD in a chest pain patient based on the patient's age, sex, and pain characteristics, known as Diamond \& Forrester method (DFM) (5). Another method is Duke Clinical Score (DCS) which considers patient's sex, age, type of chest pain, smoking, diabetes mellitus, dyslipidemia, family history of CAD and obesity (6). AHA/ACC guidelines recommend the use of DF or DCS. Pretest score: Low 0 to 8 points; Intermediate 9 to 15 points; High $>15$ points (7).

MPI performed with gated single-photon emission computed tomography (SPECT) are analyzed not only visually but also by a number of semiquantitative measures performed by the various computer programs used for imaging. Some of the important 
semiquantitative measures include Summed Stress Score (SSS), Summed Rest Score (SRS), and Summed Difference Score (SDS).The SSS, SRS, and SDS incorporate the extent and severity of perfusion defects during stress and rest. The SSS is an index obtained by adding the individual scores derived from the 17 segments that are analyzed and scored during a stress study. Each segment is scored on a 5-point scale: $0=$ normal, $1=$ mild reduction of tracer uptake (equivocal), 2 = moderate reduction of uptake (usually implies a significant abnormality), $3=$ severe reduction of uptake, $4=$ absence of uptake (8).

\section{PATIENTS AND METHODS}

A total of 89 patients with suspected or known CAD attending the National Institute of Nuclear Medicine \& Allied Science (NINMAS) from July 2016 to June 2017 were enrolled in this study. All underwent stress-rest MPI with $99 \mathrm{~m}_{\text {Tc-sestamibi. SPECT }}$ acquisition with ECG gating at rest phase was done 45-60 minutes after injection of $25 \mathrm{mCi}$ tracer on the same day following the post-stress scan (done 15-30 minutes after stress injection of $10 \mathrm{mCi}$ of tracer). Acquisition image was done with the double-headed SPECT scintillation camera with detectors $76^{\circ}$ to each other. Low Energy All Purpose (LEAP) collimators with parallel holes were used with 1.45 zoom. A symmetric $15 \%$ energy window around the $140 \mathrm{keV}{ }^{99 \mathrm{~m}}$ Tc photo-peak was set. Data was stored in 64X64 matrices (pixel size $6.59 \mathrm{cmm}, 21-27$ slices in short axis).ECG gating was done with three limb leads and acquisition was set to eight frames per R-R interval (about $153 \mathrm{~ms} /$ frame). Patients with history of cardiogenic shock, unstable angina, unorganized thrombus in left ventricular cavity, left ventricular failure, acute myocardial infarction, pregnant and lactating mother were excluded. The participants were briefed about the details of the study procedure and radioisotope was administered maintaining internationally recommended safe procedures. MPI was done by Symbia Evo Excel dual head gamma camera. SSS was automatically generated by the software while performing MPI.

For quantitative assessment of LVEF, EDV \& ESV, 4DMSPECT v4.2 software (Invia, LLC 2007) provided by Siemens medical solutions, Inc and Toshiba Corporation was applied to process \& interprete raw GSMPI images. Perfusion data, semi quantitative scores (SSS, SRS, SDS), measurement of LV volumes, LVEF and LV wall motion were obtained from GSMPI images. Statistical analysis of data was done using IBM SPSS version 20.0.0 (IBM Corporation Software Group Somers, NY) for windows. In SPSS, data were analyzed by two stages. For analysis of agreement between PPS and SSS, Bland-Altman plots were constructed.

\section{RESULTS}

The study included 89 patients ( 80 males, 9 females) with mean age $53.17 \pm 10.5$ years (range 25-81 years), mean height $1.67 \pm 0.07 \mathrm{~m}$, mean weight $69.95 \pm 9.32 \mathrm{~kg}$ and mean BMI $25.12 \pm 3.13 \mathrm{~kg} / \mathrm{m} 2$. Majority of the patients had history of typical angina (typical angina: $55.1 \%$, atypical angina: $23.6 \%$, nonangina: $21.3 \%)$. majority of patients, $(93.3 \%)$ were hypertensive, more than half $(53.9 \%)$ had type II diabetes mellitus and $82 \%$ had dyslipidemia. $55.1 \%$ patients had positive family history of CAD and $41.6 \%$ subjects were current smoker. Mean PPS was found $14.73 \pm 3.35$ and mean SSS was $16 \pm 14.01$.

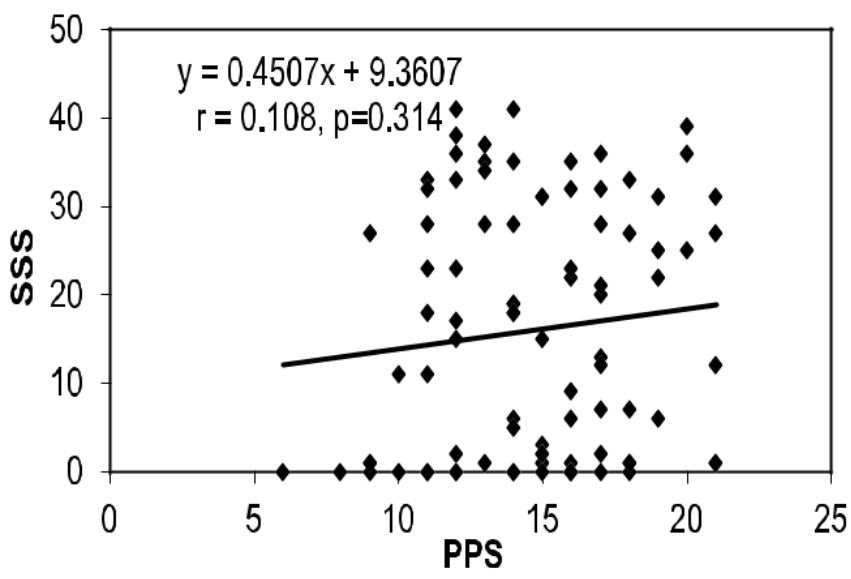

Figure 1: Scatter diagram showing positive correlation $(r=0.108 ; p=0.312)$ between PPS and SSS. 


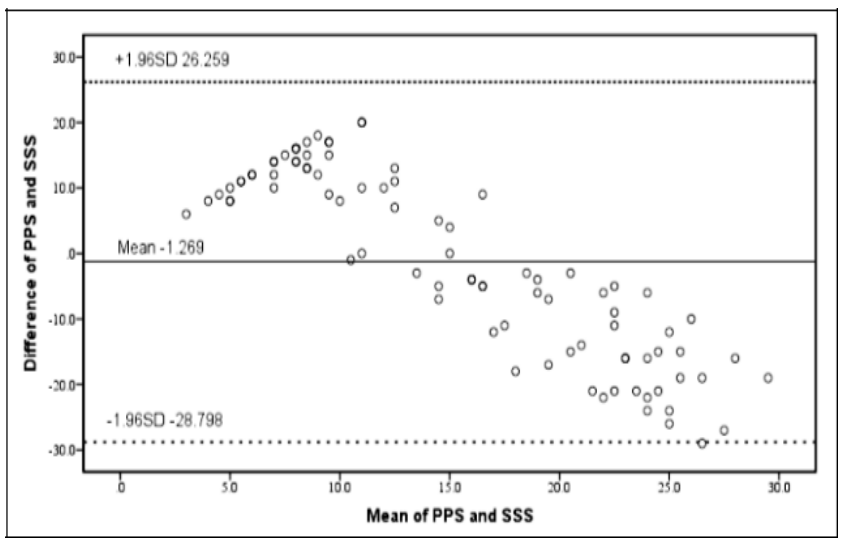

Figure 2 : Bland - Altman plot of PPS versus SSS showing good agreement between the groups.

There was a positive correlation $(\mathrm{r}=0.108 ; \mathrm{p}=$ 0.312 ) between PPS and SSS (Figure 1). According to the Bland-Altman plot, mean difference of PPS and SSS was $-1.27 \pm 14.045$. $95 \%$ of differences were found in between -28.798 and 26.259 (Figure 2).

\section{DISCUSSION}

It was observed that majority (38.2\%) of patients belonged to 51-60 years and mean age was found 53.17 \pm 10.5 years. Bittencourt et. al. found the mean age $56 \pm 13$ years, which closely resembles with the present study. It was observed that almost ninety $(89.9 \%)$ patients were male and $10.1 \%$ patients were female and male female ratio was 9:1. Bittencourt et al. and Genders et al. found $57 \%$ and $68.9 \%$ being male respectively $(9,10)$, which also closely resembles with the present study. Similar observation regarding the male predominance was also observed by Schenker et al. (11).

In this current study, mean BMI was observed $25.12 \pm$ $3.13 \mathrm{~kg} / \mathrm{m}^{2}$ which ranged from 17.3 to $41.9 \mathrm{~kg} / \mathrm{m}^{2}$. Schenker et al. found higher BMI $31.9 \pm 8.7 \mathrm{~kg} / \mathrm{m}^{2}$, than the current study and western structure and higher body surface area might explain this. History of angina was observed in more than half (55.10) of the patients and among them $23.6 \%$ had atypical angina and $21.3 \%$ had no history of angina. Schenker et al. Found 25.4\% atypical angina, $20.6 \%$ patients had typical angina nad $52.0 \%$ had no history of angina (11).
The traditional risk factors for CAD are high LDL cholesterol, low HDL cholesterol, high blood pressure, family history, diabetes, smoking and being postmenopausal for women and obesity can also be a risk factor (12). In this study, it was observed that most (93.3\%) of the patients had hypertension, $82.0 \%$ patients had dyslipidemia, $55.1 \%$ had positive family history of CAD, $53.9 \%$ diabetes mellitus (DM) and $41.6 \%$ patients were smoker. Schenker et al. reported hypertension in $79.3 \%$ and diabetes mellitus in $34.6 \%$, positive family history of CAD $34.6 \%$ and smoking in $18.4 \%$ (11). Bhattacharyya et al. reported that traditional cardiovascular risk factors such as progressing age, diabetes mellitus, hypertension, dyslipidemia, smoking, and obesity are wellaccepted for their relationship with CAD (12). Smokers have an increased risk of heart attack, depending on the number of cigarettes smoked daily and the number of years they have smoked (13). More than half of the patients suffering from diabetes mellitus die from CAD; moreover, DM patients have increased risk of developing CAD compared to patients not having DM (14).

Imamura et al. reported that the SSS was the most powerful independent predictor of all ischemic cardiac events (hazard ratio 1.1, CI 1.05 - 1.11). Abnormal SSS $(>4)$ was associated with a significantly higher cardiac event rate in patients with an intermediate to high pretest probability of CAD. Segmental perfusion scores, especially SSS, were related to a significant increase in the risk for ischemic cardiac events.

In this study a positive correlation between PPS and SSS was found. With Bland and Altman analysis mean difference between the scores was small. The bias between the scores was not significant. The differences within mean $\pm 1.96 \mathrm{SD}$ were not statistically significant. Hence PPS and SSS can be used interchangeably.

\section{CONCLUSION}

This analysis of agreement between PPS and SSS can further enhance prediction of $\mathrm{CAD}$ and upgrade the 
utilization of SSS for risk stratification in CAD patients, which will influence therapeutic management of the patients and will play a major role to reduce cardiovascular mortality and morbidity.

\section{REFERENCES}

1. Hanson MA, Fareed MT, Argenio SL, Agunwamba AO, Hanson TR. Coronary artery disease. Primary Care: Clinics in Office Practice 2013;40(1):1-6. doi:

10.1016/j.pop.2012.12.001.

2. Wong ND. Epidemiological studies of CHD and the evolution of preventive cardiology. Nature Reviews Cardiology 2014;11(5):276-89.

doi: 10.1038/nrcardio.2014.26.

3. World Health Organization, 2014. World Health rankings Live Longer Live Better.

4. Demarco DC, Papachristidis A, Roper D, Tsironis I, Byrne J, Alfakih K, Monaghan M. Pre-test probability risk scores and their use in contemporary management of patients with chest pain: One year stress echo cohort study. JRSM open 2015:14;6(11).

5. Diamond GA, Forrester JS. Analysis of probability as an aid in the clinical diagnosis of coronary-artery disease. NEJM 1979;300(24):1350-8.

6. Pryor DB, Harrell FE, Lee KL, Califf RM, Rosati RA. Estimating the likelihood of significant coronary artery disease. AJM 1983;75(5):771-80.

7. Morise AP, Jalisi F. Evaluation of pretest and exercise test scores to assess all-cause mortality in unselected patients presenting for exercise testing with symptoms of suspected coronary artery disease. JACC 2003;42(5):842-50.

8. Cerqueira MD, Weissman NJ, Dilsizian V, Jacobs AK, Kaul S, Laskey WK, Pennell DJ, Rumberger JA, Ryan T,
Verani MS, American Heart Association Writing Group on Myocardial Segmentation and Registration for Cardiac Imaging. Standardized myocardial segmentation and nomenclature for tomographic imaging of the heart. Circulation 2002;105(4):539-42.

9. Bittencourt MS, Hulten E, Polonsky TS, Hoffman U, Nasir K, Abbara S, Di Carli M, Blankstein R. European society of cardiology-recommended coronary artery Disease consortium Pretest Probability scores More accurately Predict Obstructive coronary Disease and cardiovascular events than the Diamond and Forrester score. Circulation 2006;134(3):201-11.

10. Genders TS, Steyerberg EW, Alkadhi H, Leschka S, Desbiolles L, Nieman K, Galema TW, Meijboom WB, Mollet NR, de Feyter PJ, Cademartiri F. A clinical prediction rule for the diagnosis of coronary artery disease: validation, updating, and extension. Eur Heart J2011;32(11):1316-30.

11. Schenker MP, Dorbala S, Hong EC, Rybicki FJ, Hachamovitch R, Kwong RY, Di Carli MF. Interrelation of coronary calcification, myocardial ischemia, and outcomes in patients with intermediate likelihood of coronary artery disease. Circulation 2008;117(13):1693-700.

12. Bhattacharyya PJ, Vijapur S, Bhattacharyya AK. A Study of cardiovascular risk factors correlation with the angiographic severity of coronary artery disease using Syntax score. IOSR-JDMS2016;15(1):21-28.

13. Mallaina $\mathrm{P}$, Lionis $\mathrm{C}$, Rol H, Imperiali R, Burgess A, Nixon M, Malvestiti FM. Smoking cessation and the risk of cardiovascular disease outcomes predicted from established risk scores: results of the Cardiovascular Risk Assessment among Smokers in Primary Care in Europe (CV-ASPIRE) study. BMC Public Health. 20131;13(1):362.

14. Shah BS, Deshpande SS. Assessment of demographics, treatment strategies, and evidence-based medicine use among diabetic and non-diabetic patients with acute coronary syndrome: A cohort study. JPP2014;5(2):139. 